\title{
Do We Practice What We Preach? A Review of Actual Clinical Practice with Regards to Preconception Care Guidelines
}

\author{
Michele Curtis - Steve Abelman - Jay Schulkin • \\ Jennifer L. Williams • Elizabeth M. Fassett
}

Received: 14 November 2005 / Accepted: 27 April 2006 / Published online: 29 July 2006

(C) Springer Science + Business Media, Inc. 2006

\begin{abstract}
Objectives: To review what past studies have found with regard to existing clinical practices and approaches to providing preconception care. Methods: A literature review between 1966 and September 2005 was performed using Medline. Key words included preconception care, preconception counseling, preconception surveys, practice patterns, pregnancy outcomes, prepregnancy planning, and prepregnancy surveys. Results: There are no current national recommendations that fully address preconception care; as a result, there is wide variability in what is provided clinically under the rubric of preconception care. Conclusions: In 2005, the Centers for Disease Control and Prevention sponsored a national summit regarding preconception care and efforts are underway to develop a uniform set of national recommendations and guidelines for preconception care. Understanding how preconception care is presently incorporated and manifested in current medical practices should help in the development of these national guidelines. Knowing where, how, and why some specific preconception recommendations have been successfully
\end{abstract}

\section{Curtis $(\square)$}

University of Texas-Houston,

Houston, TX

\section{S. Abelman}

March of Dimes Birth Defects Foundation,

1275 Mamaroneck Avenuse, White Plains, NY

J. Schulkin

Department of Research, American College of Obsetricians and

Gynecologists,

409 12th St., S.W., P.O. Box 96920, Washington, DC, 20090-6920

J. L. Williams · E. M. Fassett

National Center on Birth Defects and Developmental Disabilities, Centers for Disease Control and Prevention,

1600 Clifton Rd, Atlanta, GA, 30333 adopted and translated into clinical practice, as well as barriers to implementation of other recommendations or guidelines, is vitally important in developing an overarching set of national guidelines. Ultimately, the success of these recommendations rests on their ability to influence and shape women's health policy.

Keywords Preconception care $\cdot$ Preconception counseling $\cdot$ Preconception surveys $\cdot$ Practice patterns . Pregnancy outcomes · Prepregnancy planning . Prepregnancy surveys

There is a strong body of evidence to demonstrate that preconception care can modify behavioral, medical, and other health risk factors known to impact pregnancy outcomes. For example, preconception care efforts and interventions have been shown to improve folate status among women planning to conceive and to reduce the risk of fetal alcohol syndrome $[1,2]$. Controlling known medical problems prior to pregnancy not only helps to optimize the mother's health, it may also improve neonatal and pediatric outcomes [3]. Preconception care efforts and interventions have been shown to lower the risk for both major and minor birth defects among the children of women with pre-existing diabetes [4]. Women with epilepsy and their offspring benefit from preconception care counseling that manages medications, optimizes seizure control and prescribes folic acid for neural tube defect (NTD) prevention $[5,6]$.

While there is a burgeoning knowledge base on how to improve pregnancy outcomes, there are few studies examining the effects of this knowledge base on actual practice patterns. It is not well known to what extent health care providers are translating preconception care knowledge into practice for all women capable of becoming pregnant (whether planning pregnancy or not). 


\section{Material and methods}

A literature review between 1966 and September 2005 was performed using Medline to examine the impact of preconception care trials and recommendations on the clinical practice patterns of health care providers. Key words included preconception care, preconception counseling, preconception surveys, practice patterns, pregnancy outcomes, prepregnancy planning, and prepregnancy surveys. Relevant review articles regarding preconception care were also included. All studies had to be done in the United States. Studies that examined existing clinical practices and approaches directly to providing preconception care were eligible for inclusion. Studies that used indirect methods of measurement, e.g., interviews with women as a means of assessing preconception care practices of providers, were not included. A total of 11 studies were located and included analyses of practice patterns of various health care providers and specialties (see Appendix).

\section{Results}

Studies researching health care provider awareness, knowledge and practices regarding preconception care in the United States are infrequently performed, so it is difficult to fully assess health care provider approaches and practices related to preconception care. A small pilot study involving family practice nurse practitioners was done in 1987 to determine what preconception care issues were discussed with women of childbearing age during a well woman visit. Audiotapes of the visits were compared to an investigatordeveloped model for preconception counseling. Based on the results, the authors concluded that current obstetrical care had not expanded to include preconception counseling [7].

A study among family physicians conducted in 1991 using the Comprehensive Prevention Knowledge and Applications Survey Instrument found that only $37 \%$ of providers reported counseling women of childbearing age about preconception behaviors $>75 \%$ of the time [8]. When asked how often they felt ready to counsel women on preconceptional factors, about $70 \%$ of providers said they were prepared at least three-fourths of the time. However, perceived preparedness did not equate to counseling success. Among providers who did counsel, only about one-third reported being successful at helping their patients change preconceptional behaviors at least $75 \%$ of the time.

A 1991 study conducted among family practice and internal medicine residents in an inner-city public hospital found that both groups of residents reported low levels of knowledge and management decision skills regarding preconception care when compared with standard recommendations, despite their favorable attitudes towards preconception care [9]. In this survey, close to $50 \%$ of the residents did not mention family planning during counseling sessions with women of reproductive age and $74 \%$ would not raise the issue of congenital defects in their diabetic patients seeking to become pregnant. While both family practice and internal medicine residents had favorable attitudes towards preconception care, the family practice residents scored higher in positive attitude about preconception care. There was no difference between the groups in terms of management skills, and there was no sustained difference between groups in the area of knowledge. Previous rotation through a high-risk perinatal clinic increased scores in areas of management and knowledge compared to family practice or internal medicine residents who had not rotated through such a clinic, but these differences were not significant [9].

In an effort to assess the practices of genetic counseling and screening for consanguineous couples, their pregnancies, and their children, 1,582 surveys were mailed to board certified genetic counselors and medical geneticists in the United States in 1999 [10]. While the response rate was very poor (only 20\%) there was wide variability in suggested screening practices for consanguineous couples before conception, during pregnancy, after birth, and for children placed for adoption. Respondents seemed to generally agree to do screening based on ethnicity but there was no consistency regarding which genetic disorders to include in these screening efforts.

A mailed survey of obstetrician-gynecologists in 2000 focused on issues around folate for the prevention of NTDs [11]. Almost 97\% of participants knew that when taken early enough, folic acid reduced the incidence of NTDs. While two-thirds of respondents said they routinely screened their pregnant patients for folate intake, only 53\% screened nonpregnant women of childbearing age. The authors of the study concluded that most obstetrician/gynecologists are aware of the link between folate intake and NTDs although the data showed that physicians who routinely screened for folate intake correctly answered more survey questions than those who did not.

As part of a larger effort to improve the documentation and delivery of preconception care, Bernstein and colleagues evaluated the knowledge base and awareness of preconception care for women in their childbearing years seeking care in an inner city outpatient gynecology clinic in 2000 [12]. Providers in the clinic included physicians and nurse practitioners. A pre-intervention chart review found that many of the providers were not addressing family planning services, domestic violence, nutrition and medical risk factors, medication use, appropriate counseling and use of referral services during gynecologic visits. The authors concluded that the providers did not take the opportunity to discuss preconception care during routine gynecologic visits. 
A series of studies looking at provider knowledge and practice regarding preconception and prenatal screening for cystic fibrosis (CF) was conducted during 2001-2004 using the Collaborative Ambulatory Research Network (CARN) [13]. Results showed that almost one-half of the responders did not ask nonpregnant women of childbearing age about their family history of $\mathrm{CF}$, nor did they provide information on CF screening. Close to $90 \%$ of respondents did offer CF screening or inquire about a family history of CF among pregnant women. Of those Ob/Gyns who did selectively screen for $\mathrm{CF}$ among their pregnant patients, about $25 \%$ utilized all of the selection criteria noted in the American College of Obstetricians and Gynecologists (ACOG) recommendations.

In 2002 and 2003 the March of Dimes examined folic acid knowledge and practice patterns of health care providers in obstetric/gynecology and family/general practice settings [14]. Survey results found that, while knowledge about the need for supplementation and timing of folic acid was high, increased knowledge about unintended pregnancy rates and correct doses of folic acid for prevention of occurrence and recurrence of NTDs is needed. A little more than half of all providers knew the correct dose of folic acid $(400 \mathrm{mcg}$ daily) for the prevention of NTDs. This percentage dropped dramatically when providers were asked the correct dose of folic acid for a woman with a history of a previous NTDaffected pregnancy. Almost two-thirds of providers did not know the folic acid dose for recurrence prevention (4 mg daily).

In the surveys, the vast majority of providers reported always recommending folic acid supplementation for women who expressed interest in becoming pregnant, approximately $60 \%$ of respondents who provide prenatal care reported seeing less than one fifth of their prenatal patients for a preconception care visit [14]. At annual gynecologic or wellwoman examinations as well as other types of patient visits, less than $60 \%$ of respondents always or usually addressed supplementation. Lack of knowledge (39\%) and lack of time (30\%) during a busy exam schedule were the most cited reasons providers gave for not always recommending folic acid.

In 2004 a survey was sent to Florida health care providers to establish their baseline knowledge and practice behavior regarding folic acid and NTDs [15]. At baseline, 97\% of providers reported awareness of the protective nature of folic acid during preconception and early in the first trimester; however, less than half of providers reported that they discuss folic acid consumption with all women of child-bearing age. After a statewide educational program, the same providers were surveyed again to see if there had been any change in knowledge base or practice patterns. The survey done after the educational outreach efforts did demonstrate an increase in awareness and an increase in the percentage of providers who recommended peri-conceptional use of folic acid to prevent NTDs. The authors note, however, that despite these modest gains, the need for continued education and modification of provider practice patterns remains.

A recent survey by ACOG was done to assess the opinions and practices of obstetrician-gynecologists regarding preconception care, and their perception of patients' receptiveness to preconception services [16]. The vast majority of physicians $(97.3 \%)$ stated that they provide preconception care for their patients, although obstetrician-gynecologists were more likely to do so than providers who only practiced gynecology. The majority of physicians (87\%) agreed with the definition of preconception care as "Specialized prepregnancy care that focuses on issues not typically addressed during a routine exam which are specific to ensuring an optimal pregnancy outcome." Most of the respondents indicated that women are more likely to present for preconception care to ensure a healthy pregnancy than because of an elevated risk for a birth defect or developmental disorder. The majority of physicians agreed that preconception care is an important issue and that it has a positive effect on pregnancy outcomes, though only $21 \%$ agreed that it was a high priority in their workload. Half of the physicians said there was not enough time to provide preconception care to all women with reproductive potential, nor were there reimbursement incentives to do so.

\section{Discussion}

Preconception care- care that is initiated before pregnancyis advocated to help women reduce their risk for adverse pregnancy outcomes and make informed decisions regarding their readiness for and timing of pregnancy [17]. Appropriate preconception care improves pregnancy outcomes and has several components. These include, but are not limited to: the systematic identification of preconceptional risks through an assessment of the woman's reproductive, family, and medical history; the family and medical history of the father; the woman's nutritional status, social concerns, and any drug or substance exposures she or the father may have (had); discussions regarding possible effects of any existing medical problems and potential interventions; screening for infectious diseases with treatment and immunization where indicated; discussions about environmental exposures, both occupational and household; a review of the circumstances of the woman's life and behavioral patterns; counseling and discussion about birth spacing including real and perceived barriers to achieving her desires; and inquiry and education regarding contraceptive use.

Traditionally, preconception care endeavors have focused on women who have a chronic medical condition, a history of a poor outcome in a previous pregnancy, or who are 
planning to become pregnant in the near future. For women with chronic medical conditions, the effect(s) of their illness(es) range from minimal to limited activity to hospitalization. Drawing on information for the 1996 National Health Interview Survey (NHIS), 9.9\% of women ages 18-44 years had some limitations placed on their activity levels due to chronic disease [18]. There is clear evidence that the initiation of preconception counseling and care for women with some chronic health conditions, e.g., diabetes, will positively impact pregnancy outcomes [19]. For women with a history of a poor pregnancy outcome in the past, pre- or interconceptional intervention strategies have been demonstrated to be effective [20]. Limiting preconception care endeavors to women actively planning pregnancy, however, reaches a limited audience as almost $50 \%$ of pregnancies in the U.S. are unintended [21]. Comprehensive preconception care, however, encompasses much more than just these risk categories and is inclusive of all women with the potential to become pregnant.

Women of childbearing age visit their physician an average of about three times per year; these visits represent opportunities to deliver preconception care and messages related to preconception care [22]. In reviewing the literature related to the clinical practice of preconception care, it is clear that most of these opportunities are either missed or foregone. In an article focusing on preconceptional health promotion, Moos proposes 5 categorical reasons for why providers are inconsistent in applying proven prevention strategies for poor pregnancy outcomes: lack of knowledge regarding the incidence of unintended pregnancy, inadequate provider education, lack of confidence that preconceptional health counseling is valuable, a belief that women will 'know' to seek the care appropriate to their needs, and concerns over lack of reimbursement coverage for preconceptional visits [23].

Changing behavior patterns is no small feat, but understanding the 'hows' and 'whys' of change(s) in provider practice patterns is becoming better understood. For example, in diffusion theory, diffusion is the process through which an innovation is adopted for use or application by a community [24]. One proposed method for facilitating diffusion of innovation utilizes an expert opinion leader process whereby a designated (or identified) local physician leader communicates information about new innovation(s) or knowledge to colleagues and then follows up with them about their personal experiences with the information [25]. This approach has demonstrated effectiveness for eliciting changes in practice patterns in certain situations and settings, but it is not uniformly effective in all instances [26-29].

In his assessment of how research findings get translated into "best practices", Green postulates that there are three areas representing barriers to the adoption of best practice: 1) accessibility gap, i.e., practitioners do not have the same resources available as the researchers; 2) credibility gap, e.g., a comparison of differences in practice settings or populations that might 'explain' why the research is not applicable to the general practitioner; 3 ) expectations gap, e.g., the practitioner views the research findings as unnecessary goals to set for their own practices [30]. These barriers are not insurmountable, and there is evidence that different approaches to changing provider behavior patterns are effective [26, 27]. In an article by Cullum, several model programs are reviewed that have demonstrated changes in the practices of health care providers related to preconception care [31].

Studies evaluating effective preconception care interventions and strategies are numerous, and continue to grow. There is also an increasing amount of information about how patients assimilate and act upon recommendations regarding preconception care. There is little data, however, that analyzes the impact and integration of preconception care innovations on daily clinical practice(s). One limitation of this literature review is that articles with secondary goals of evaluating actual clinical practices may have been overlooked. Efforts were made to include all relevant search terms but it is possible that articles written before 1985 may have been inadvertently excluded if different keywords were used at that time. Nonetheless, there is a large need for further research into the contrast between the science of preconception care and the reality of actual clinical practice.

\section{Conclusion}

Preconception care seeks to promote the health of women of childbearing age prior to conception and to improve pregnancy-related outcome. Various authors propose the integration of preconception care into routine wellness care for all women of reproductive potential [23, 32-34]. The question is how do we raise the level of importance and prioritization of preconception care during individual encounters as well as on a national health policy level? Proving the efficacy of a practice does not guarantee an actual change in practice and simply prompting providers to incorporate preconception care will not suffice. Creating the expectation that preconception care is an ongoing process during a woman's reproductive life span will require a myriad of efforts concurrently directed at providers, patients, payors, and policy makers.

In 2005, the Centers for Disease Control and Prevention sponsored a national summit regarding preconception care with a broad cross-section of stakeholders. Efforts are currently underway to develop a uniform set of national recommendations and guidelines for preconception care. Understanding how preconception care is presently incorporated and manifested in current medical practices should help in the development of these national guidelines. Knowing where, how, and why some specific preconception 
recommendations have been successfully adopted and translated into clinical practice, as well as barriers to implementation of other recommendations or guidelines, is vitally important in developing an overarching set of national guidelines. Ultimately, the success of these recommendations rests on their ability to influence and shape women's health policy.

\section{Appendix}

Articles Identified in Search Analyzing Provider Practices in Preconception Care

1. Barron ML, Ganong LH, Brown M. An examination of preconception health teaching by nurse practitioners. $\mathrm{J}$ Adv Nurs 1987 Sep;12(5):605-10.

2. Bennett RL, Hudgins L, Smith CO, Motulsky AG. Inconsistencies in genetic counseling and screening for consanguineous couples and their offspring: the need for practice guidelines. Genet Med 1999 Sep-Oct; 1(6):28692

3. Bernstein PS, Sanghvi T, Merkatz IR. Improving preconception care. J Reprod Med 2000 Jul;45(7):546-52.

4. Cullum AS. Changing provider practices to enhance preconceptional wellness. J Obstet Gynecol Neonatal Nurs 2003 Jul-Aug;32(4):543-9.

5. Cullum AS. Changing provider practices to enhance preconceptional wellness. J Obstet Gynecol Neonatal Nurs 2003 Jul-Aug;32(4):543-9.

6. Freda MC, Chazotte C, Bernstein P, Harrison E. March of Dimes Preconception Working Group. Interdisciplinary development of a preconception health curriculum for four medical specialties. Obstet Gynecol 2002 Feb;99(2):301-6.

7. Hauser KW, Lilly CM, Frias JL. Florida health care providers' knowledge of folic acid for the prevention of neural tube defects. South Med J 2004 May;97(5):4379.

8. Jennifer Williams, MSN, MPH APRN-BC 1, Steve Abelman, MBA2, Elizabeth Fassett, MS, CHES1, Cheryl Stone, MSPH3, Joanne Petrini, PhD2, Karla Damus, PhD, RN2, Joseph Mulinare, MD, MSPH1. (National Center on Birth Defects and Developmental Disabilities, Centers for Disease Control and Prevention1, March of Dimes2, Rynne Marketing3) Health Care Provider Knowledge and Practices Regarding Folic Acid, United States, 2002-2003. Maternal Child Health. In print- it will be in the special supplement this paper is in.

9. Morgan MA, Driscoll DA, Mennuti MT, Schulkin J. Practice patterns of obstetrician-gynecologists regarding preconception and prenatal screening for cystic fibrosis. Genet Med 2004 Sep-Oct;6(5):450-5.
10. Morgan MA, Hawks D, Zinberg S, Schulkin J. What obstetrician-gynecologists think of preconception care. Maternal Child Health. In print (this issue).

11. Moser R Jr, McCance KL, Smith KR. Results of a national survey of physicians' knowledge and application of prevention capabilities. Am J Prev Med 1991 NovDec;7(6):384-90.

12. Power ML, Holzman GB, Schulkin J. Knowledge and clinical practice regarding folic acid among obstetrician-gynecologists. Obstet Gynecol 2000 Jun;95(6 Pt 1):895-8.

\section{References}

1. de Weerd S, Thomas CMG, Cikot RJLM, Steegers-Theunissen RPM, de Boo TM, Steegers EAP. Preconception counseling improves folate status of women planning pregnancy. Obstet Gynecol 2002;99:45-50.

2. MMWR May 16, 2003;52(19):441-444.

3. Haas JS, Fuentes-Afflick EF, Stewart AL, Jackson RA, Dean ML, Brawarsky P, Escobar GJ. Prepregnancy health status and the risk of preterm delivery. Arch Pediatr Adolesc Med 2005;159:58-63.

4. Ray JG, O'Brien TE, Chan WS. Preconception care and the risk of congenital anomalies in the offspring of women with diabetes mellitus: a meta-analysis. QJM; 2001.

5. Brundage SC. Preconception health care. Am Fam Physician 2002;65(12):2507-14.

6. Betts T, Fox C. Proactive pre-conception counseling for women with epilepsy-is it effective? Seizure 1999;8:322-7.

7. Barron ML, Ganong LH, Brown M. An examination of preconception health teaching by nurse practitioners. J Adv Nurs 1987 Sep;12(5):605-10.

8. Moser R, McCance KL, Smith KR. Results of a national survey of physicians' knowledge and application of prevention capabilities. Am J Prev Med 1991;7:384-90.

9. Conway T, Hu TC, Mason E, Mueller C. Are primary care residents adequately prepared to care for women of reproductive age? Fam Plann Perspect 1995;2:66-70.

10. Bennett RL, Hudgins L, Smith CO, Motulsky AG. Inconsistencies in genetic counseling and screening for consanguineous couples and their offspring: the need for practice guidelines. Genet Med 1999 Sep-Oct;1(6):286-92.

11. Power ML, Holzman GB, Schulkin J. Knowledge and clinical practice regarding folic acid among obstetrician-gynecologists. Obstet Gynecol 2000;(6 Pt. 1):895-8.

12. Bernstein PS, Sanghvi T, Merkatz IR. Improving preconception care. J Reprod Med 2000 Jul;45(7):546-52.

13. Morgan MA, Driscoll DA, Mennuti MT, Schulkin J. Practice patterns of obstetrician-gynecologists regarding preconception and prenatal screening for cystic fibrosis. Genet Med 2004;6:450-5.

14. Williams J, Abelman S, Fassett E, Stone C, Petrini J, Damus K, Mulinare J. Marketing Health Care Provider Knowledge and Practices Regarding Folic Acid, United States, 2002-2003. Maternal Child Health 2006. In print. (Accompanying article in this supplement).

15. Hauser KW, Lilly CM, Frias JL. Florida health care providers' knowledge of folic acid for the prevention of neural tube defects. South Med J 2004;97(5):437-9.

16. Morgan MA, Hawks D, Zinberg S, Schulkin J. What obstetriciangynecologists think of preconception care. Maternal Child Health 2006. In print (Accompanying article in this supplement). 
17. Jack BW, Culpepper L. Preconception care: risk reduction and health promotion in preparation for pregnancy. JAMA 1990;264:1147-9.

18. Adams P, Hendershot G, Marano M. Current estimates from the National Health Interview Survey, 1996. Vital Health Statistics 1999;10:83-4.

19. Dicker D, Feldberg D, Samuel N, et al. Spontaneous abortion in patients with insulin-dependent diabetes mellitus: the effect of preconceptional diabetic control. Am J Obstet Gynecol 1988;158:1161-4.

20. Biermann J, Dunlop A, Brady C, Dubin CS, Brann A. Promising Practices in Preconception Care for Women at Risk for Poor Health and Pregnancy Outcomes. Mat Child Health 2006. In print. (Accompanying article in this supplement).

21. Henshaw SK. Unintended pregnancy in the United States. Fam Plan Perspecti 1998;30:24-9.

22. Cherry DK, Woodwell DA. National Ambulatory Medical Care Survey: 2000 summary. Adv Data 2002;1-32.

23. Moos MK. Preconceptional Health Promotion: Progress in changing a prevention paradigm. J Perinat Neonat Nurs 2004;18:2-13.

24. Rogers EM. Diffusion of innovations (4th ed). New York: Free Press; 1995.

25. Greer AL. The state of the art versus the state of the science. The diffusion of new medical technologies into practice. Int $\mathrm{J}$ Technol Assess Health Care. 1988;4(1):5-26.

26. Oxman AD, Thomson MA, Davis DA, et al. No magic bullets: a systematic review of 102 trials of interventions to improve professional practice. Can Med Assoc J 1995;153:1423-31.
27. Bero LA, Grilli R, Grimshaw JM, et al. Closing the gap between research and practice: an overview of systematic reviews of interventions to promote the implementation of research findings. BMJ 1998;317:465-8.

28. Lomas J, Enkin M, Anderson GM, et al. Opinion leaders vs. audit and feedback to implement practice guidelines: delivery after previous cesarean section. JAMA 1991;265:2202-7.

29. Thomson MA, Oxman AD, Haynes RB, et al. Local opinion leaders to improve health professionals practice and health care outcomes. In: The Cochrane library. Oxford, UK: Update Software, 1999.

30. Green L. From research to "best practices" in other settings and populations. Am J Health Behav 2001;25:165-78.

31. Cullum AS. Changing Provider Practices to Enhance Preconceptional Wellness. JOGNN 2003;32:543-9.

32. Misra DP, Grason H, Weisman C. An Intersection of Women's and Perinatal Health: The Role of Chronic Conditions. Women's Health Issues 2000;10:256-67.

33. Besculides M, Laraque F. Racial and ethnic disparities in perinatal mortality: applying the perinatal periods of risk model to identify areas for intervention. J Natl Med Assoc 2005 Aug;97(8):112832.

34. Haas JS, Fuentes-Afflick E, Stewart AL, Jackson RA, Dean ML, Brawarsky P, Escobar GJ. Prepregnancy health status and the risk of preterm delivery. Arch Pediatr Adolesc Med 2005 Jan;159(1):5863. 